\title{
Phase Diagram of Magnetization Reversal Processes in Nanorings
}

\author{
Wen Zhang and Stephan Haa:* \\ Department of Physics and Astronomy, University of Southern California, Los Angeles, CA 90089, USA
}

\begin{abstract}
We investigate magnetization reversal processes of magnetic nanorings. Using a recently developed efficient cartesian coordinates fast multipole method, magnetization switching phase diagrams are constructed for such structures. We find that the dominant switching mechanisms strongly depend on the relative spatial dimensions of the rings, i.e. height, width and radius. Furthermore, they are found to depend on a characteristic exchange length scale, which is determined by the competing short-range ferromagnetic and long-range dipolar interactions in these systems. Detailed simulations allow us to identify three novel switching mechanisms, which should be useful for building high density storage systems.
\end{abstract}

\section{INTRODUCTION}

Mesoscopic magnetic elements have attracted tremendous attention during the last two decades $\stackrel{1,2,3}{ \pm}$ In particular, there has been an upsurge of interest in small magnetic rings during the last few years $\stackrel{4,5}{\underline{4}}$ These systems exhibit many novel physical phenomena with promise for applications, such as high density data storage ${ }^{6.7 .8}$, magnetic logics $\frac{9}{}$ and biomedical sensoring ${ }^{10,11}$. Furthermore, nanoring geometries have proven to be a fertile platform for the investigation of fundamental physical questions concerning domain walls. 12 One of the most useful properties of magnetic rings is that they can stabilize flux-closed vortex states. These vortex states are free of stray fields and largely insensitive to edge imperfections, thus resulting in highly controllable magnetization reversal processes. Vortex states have been found to be stable in rings with diameters as small as $10 \mathrm{~nm}$. In contrast, vortex states in magnetic discs exist only when their size is fairly large, i.e. with diameters larger than $100 \mathrm{~nm}$, due to the existence of vortex core regions, which lead to high energy penalties. 13

Experimentally, nanomagnetic rings have been produced with a wide range of spatial dimensions, extending from dozens of micrometers down to nanometer scales 14 . Various states have been observed to be stable or metastable at remanence, i.e. in the absence of an applied magnetic field, including the vortex state (V), the onion state $(\mathrm{O})$, i.e. two ferromagnetic domains separated by domain walls at opposing ends of the ring structure, and the twisted (or saddle) state $(\mathrm{T})^{15}$ ), i.e. a vortex state interrupted by a $360^{\circ}$ domain wall. Several works have been devoted to determining the phase diagram for nanoring structures $13,16,17,18$. Apart from the phase diagram at remanance, magnetization reversal processes driven by external magnetic fields are another important characteristic property of magnetic elements. Several switching processes have already been discovered $^{19}$. Experimentally, switching phase diagrams have been constructed for micron-scale rings. 20 However, the properties of nano-scale rings are still under investigation, and a number of new features have recently been discovered $21,22,23$

In this paper, we identify several switching processes, which according to our knowledge have not yet been reported in the literature, and construct switching phase diagrams for magnetic nanorings. We use Monte Carlo simulations combined with a recently developed efficient cartesian coordinates fast multipole technique $24,25,26,27$ to analyze the magnetization reversal processes for various nanoring dimensions. Neglecting the crystalline energy, the total energy $(E)$ of a magnetic nanoparticle in a magnetic field consists of three terms: the exchange interaction, the dipolar interaction, and the Zeeman energy,

$$
\begin{aligned}
E= & -J \sum_{<i, j>} \vec{S}_{i} \cdot \vec{S}_{j}+D \sum_{i, j} \frac{\vec{S}_{i} \cdot \vec{S}_{j}-3\left(\vec{S}_{i} \cdot \hat{r}_{i j}\right)\left(\vec{S}_{j} \cdot \hat{r}_{i j}\right)}{r_{i j}^{3}} \\
& -\vec{H} \cdot \sum_{i} \vec{S}_{i} .
\end{aligned}
$$

Here $J>0$ is the ferromagnetic exchange constant, which is assumed to be non-zero only for nearest neighbors, $D$ is the dipolar coupling parameter, and $\vec{r}_{i j}$ is the displacement vector between lattice sites $i$ and $j$. Note that $\vec{S}$ is a dimensionless unit spin vector with magnetic moments $\vec{\mu}=|\mu| \vec{S}$.

Experimentally, the most studied magnetic nanomaterials are made of Co, Fe, permalloy and supermalloy. For these materials, the ratio $D / J a^{3}$ falls into the range between $10^{-3}$ and $10^{-5}$, where $a \simeq 0.3 \mathrm{~nm}$ is the lattice constant. In the Monte Carlo simulations, single spin updates are adopted to yield quasi time dependent behavior 28 We choose $J a^{3} / D=5000$ which is close to the parameters of Co. Since we are trying to discover the generic property of nanomagnets, the absolute values of parameters used in this paper are not as important as their relative magnitudes. To state our conclusions in the most general terms, we define an exchange length,

$$
L_{e x}=\alpha a \sqrt{J a^{3} / D}
$$

whose overall prefactor $\alpha$ may vary between 0.5 and 2 , depending on the material. Here we choose $\alpha=1$, and assume a typical value $a=0.3 \mathrm{~nm}$, such that $L_{e x} \sim 20$ $\mathrm{nm}$. This exchange length is very important in determining the properties of nanomagnetic systems. It is a measure of the spatial extent of magnetic domains. For 
instance, the typical diameter of vortex cores has been shown to be $1.3 L_{e x} .13$ The temperature for the Monte Carlo simulations is set to $50 \mathrm{~K}$, and at least $10^{4}$ Monte Carlo steps are performed for each field point. To facilitate the following discussion, we define $R_{o}$ as the outer ring radius, $R_{i}$ as the inner ring radius, $w=R_{o}-R_{i}$ as the width and $h$ as the ring height (see the inset of Fig 1(a)).

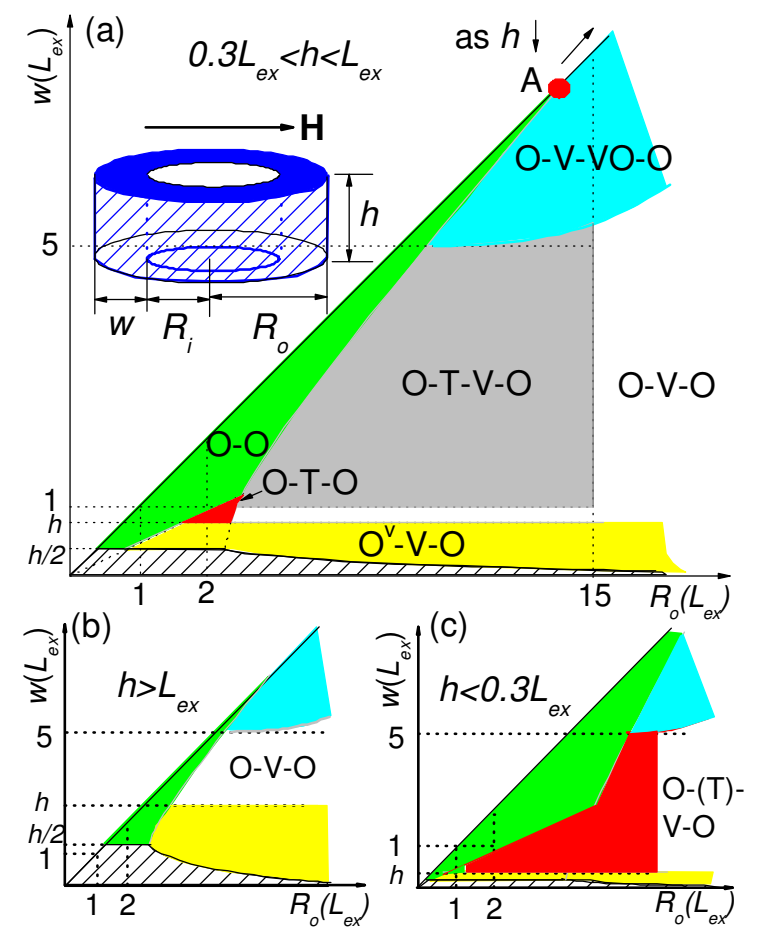

FIG. 1: (color online) Switching phase diagrams of magnetic nanorings. As indicated in the inset, $h$ denotes the ring height, $R_{i}$ and $R_{o}$ are the inner and outer ring radii, and $w=R_{o}-R_{i}$ is the width. A magnetic field $\mathbf{H}$ is applied in plane horizontally. The magnetic exchange length $L_{e x}$ is defined in the text. Phase diagrams are shown for the cases (a) $0.3 L_{e x}<h<L_{e x}$, (b) $h>L_{e x}$, (c) $h<0.3 L_{e x}$. The shaded areas in the regime of small $w$ 's represent rings whose remanence states are outof-plane ferromagnets, and hence not relevant for switching. The color filled areas represent the different switching processes discussed in the text. green: onion - onion $(\mathbf{O}-\mathbf{O})$; red: onion - twisted - onion (O-T-O); yellow: vertical onion - vortex - onion $\left(\mathbf{O}^{\mathrm{V}}-\mathbf{V}-\mathbf{O}\right)$; gray: onion - twisted - vortex - onion (O-T-V-O); cyan: onion - vortex - vortex core - onion $(\mathbf{O}-\mathbf{V}-$ VC-O); white: onion - vortex - onion $(\mathbf{O}-\mathbf{V}-\mathbf{O})$. The point 'A' indicates the onion - vortex transition for discs. This critical point moves towards larger $\left(w, R_{o}\right)$ values when $h$ decreases.

\section{SWITCHING PHASE DIAGRAMS}

We begin by summarizing our findings along with previously reported results in the switching phase diagrams shown in Fig 1. Until now, there are three dominant mag- netization reversal processes which have been intensively studied and discussed in the literature.

\section{A. Previously Reported Switching Processes}

1. The first process is one-step (or single) switching O-O, whose magnetization curve $M(H)$ is illustrated in Fig 2(b)). This is a direct onion-to-onion switching process, whereby an onion state is reversed to the opposite onion state directly via a coherent rotation, as two magnetic domain walls move in the same rotational direction. It is worth noting that when the ring radius is sufficiently small $\left(R_{o}<L_{e x}\right)$, the onion state is replaced by a single domain ferromagnetic state.

2. The second process is two-step or double switching O-V-O, shown in Fig. 2(c). Here, two opposite onion states are separated by a vortex state. This is the dominant switching process for most reported magnetic rings, stabilized by any spurious spatial asymmetry. At the first transition point, either one wall is pinned stronger than the other or both walls move towards each other due to asymmetry. In both cases the two domain walls approach each other with increasing applied magnetic field. When these two domain walls meet, they annihilate each other, provided the ring is sufficiently wide $\left(w>5 L_{e x}\right)$. If not, intermediate metastable twisted states have recently been found to exist for small narrow rings 21 . In most cases, the remanence state is an onion state. However, when the ring is sufficiently thick and wide 29,30 , the remanance state is a vortex state.

3. The third process is triple switching $\mathbf{O}-\mathbf{V}-\mathbf{V C - O}$, shown in Fig,2(d) 30,31,32 The switching mechanism is essentially the same as for $\mathbf{O}-\mathbf{V}-\mathbf{O}$, except that the vortex state does not deform into an onion state abruptly, but first nucleates a vortex core in one arm of the ring. The core then moves slowly to the outer rim. During this part of the process the state is called a "vortex core" state (VC). To some extent, this is similar to the switching process of magnetic discs, where the core in the vortex state is shifted by the magnetic field and ultimately exits at the boundary (see Fig. 2(a)).

These magnetization reversal processes are found in relatively large rings $\left(R_{0} \gg L_{e x}\right)$ with spatial dimensions in the micrometer range. However, it is reasonable to expect that in the sub-micron and truly nano ranges, new phenomena exist and wait to be explored. Therefore, we have performed simulations for a wide range of parameters and identified the following three additional magnetization reversal processes. 


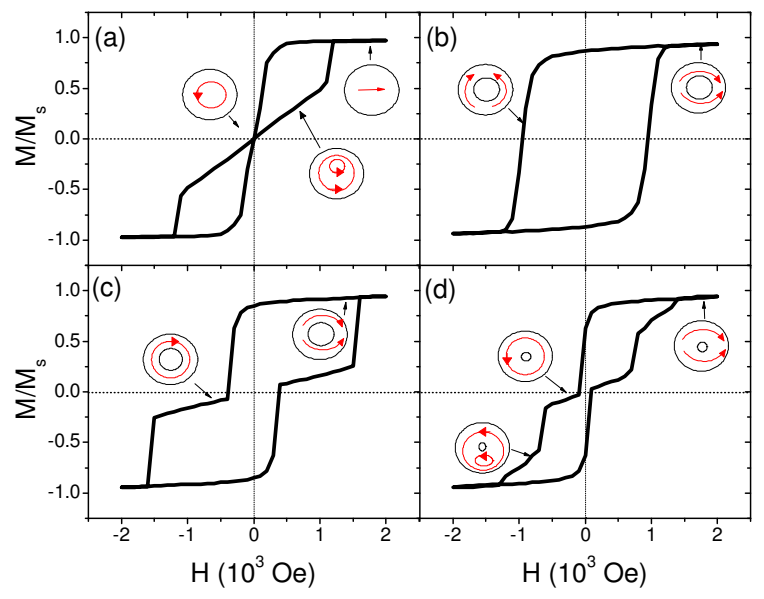

FIG. 2: (color online) Magnetic switching processes in nanoring structures and their associated hysteretic behavior. (a) hysteresis of magnetic discs $\left(R_{i} \rightarrow 0\right)$, (b) one-step O-O switching of rings, (c) double switching $\mathbf{O}-\mathbf{V}-\mathbf{O}$ of rings, (d) triple switching $\mathbf{O}-\mathbf{V}-\mathbf{V C}-\mathbf{O}$ of rings.

\section{B. Vertical Onion - Vortex - Onion Switching: $\mathrm{O}^{\mathrm{V}}-\mathrm{V}-\mathrm{O}$}

On first sight, this process appears to fall in the same category as $\mathbf{O}-\mathbf{V}-\mathbf{O}$, since its hysteresis, shown in Fig. 3, shares characteristics similar to double switching (Fig[2(c)). The switching mechanism behind it, however, is quite different. The onion state here does not consist of two transverse head-to-head domain walls, but rather of two vertical head-to-head domain walls (see the areas highlighted by open red circles in Fig. 3), where the spins point out-of-plane rather than in-plane. Hence, we call the state involving vertical head-to-head domain walls a "vertical" onion state $\left(\mathrm{O}^{\mathrm{V}}\right)$. The big blue arrow in Fig. 3 points to a small step in the magnetization curve, indicating the appearance of out-of-plane domains. The two domains may point parallel or antiparallel. However, the parallel configuration is preferred, since the switching of $\mathbf{O}-\mathbf{V}$ is more controllable and the transition is sharper compared with the antiparallel case. As we can see from Fig. 3, when the two domains point in the same direction, the $\mathbf{O}-\mathbf{V}$ transition is simply achieved by rotating spins out-of-plane in the intermediate region between the two domain walls (highlighted by the red rectangle), which subsequently align with the out-of-plane domain wall spins. In the antiparallel case, the domain wall which moves faster will first rotate to form a normal in-plane head-to-head domain wall. Then the other domain wall rotates to in-plane alignment, such that the two domain walls can easily annihilate. This second process results in a slightly larger and broader transition field, which however can be easily avoided by applying a small outof-plane magnetic field.

The $\mathbf{O}^{\mathrm{V}}-\mathbf{V}-\mathbf{O}$ process has not been reported before, as most of the initial attention in the field had been focused

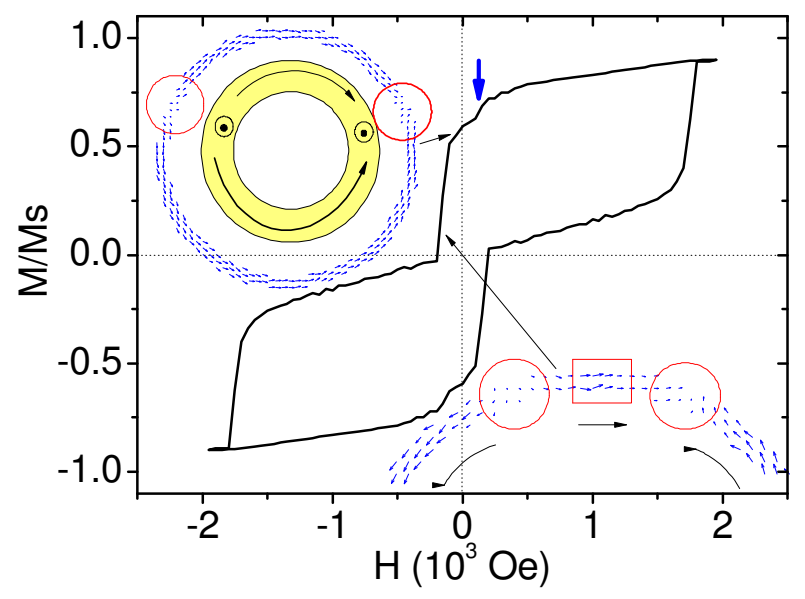

FIG. 3: (color online) Typical hysteresis curve for out-of-plane $\mathbf{O}^{\mathrm{V}}-\mathrm{V}-\mathrm{O}$ switching $\left(R_{o}=70 \mathrm{~nm}, w=7 \mathrm{~nm}, h=10 \mathrm{~nm}\right)$. The insets are the snapshot during the $\mathbf{O}^{\mathrm{V}}-\mathbf{V}$ transition. The areas with strong out-of-plane components are highlighted by open red circles. The big blue arrow points to a small step indicating the appearance of out-of-plane domains.

on thin film rings $(h \ll w)$. As seen in the phase diagrams of Fig. 11 this switching process indeed belongs to rings with $w<h$ (see the yellow regions). Shape anisotropies force the spins in the domain walls to point out-of-plane. This process is crucial when one wants to use vortex states in high-density memory applications. In the thin film case $\left(h<L_{e x}\right)$, vortex states are not easy to form when the ring radius is small (say $R_{o}<100 \mathrm{~nm}$ ). Instead, twisted states will stand in the way, preventing the formation of vortex states, which will be discussed in detail next. The only limit of this $\mathrm{O}^{\mathrm{V}}-\mathrm{V}-\mathrm{O}$ process is the single domain limit for the ring. As long as onion states exist, this scheme applies. However, when the ring is extremely small, the onion state will be replaced by a single domain state. Roughly speaking, this transition happens at $R_{o} \sim L_{e x}$. We use the remanence magnetization as an order parameter to locate this transition, as shown in Fig. 4. The onion state is characterized by a relatively small magnetization (approximately 0.68 for the chosen $w$ and $h$ ), since most of the spins are aligned along the ring boundary, whereas the single domain state is characterized by a magnetization close to saturation, since in this case nearly all the spins align in the same direction. Obviously, the transition region (shaded area in Fig. 44) is $R_{o} \approx 20-40 \mathrm{~nm} \approx 1-2 L_{e x}$. For rings with $R_{o}<L_{e x}$, the only magnetization reversal process will be one-step switching via coherent single domain rotation. Therefore, the $R_{o}$ of rings has to be at least $L_{e x}$ if the vortex state is desired. 


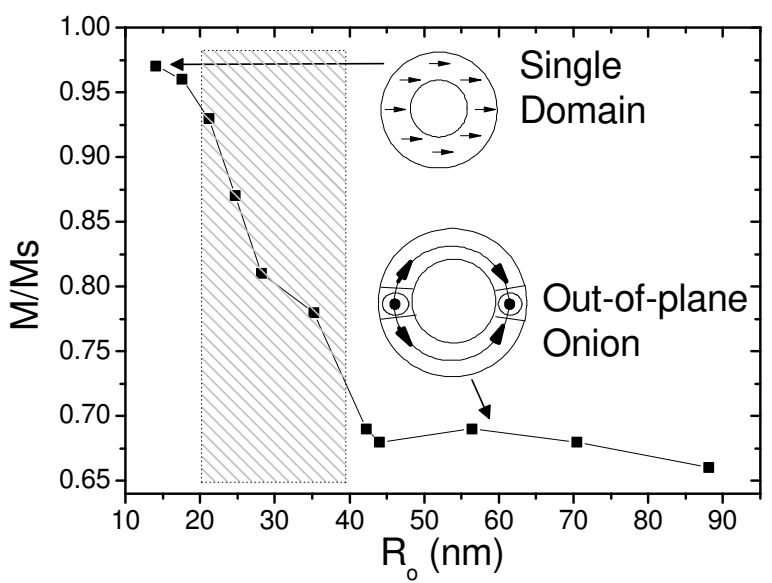

FIG. 4: (color online) Single domain to onion state transition with incrreasing ring diameter. Here, the height is fixed at $23 \mathrm{~nm}$, and the width is fixed at $15 \mathrm{~nm}$. The shaded area represents the transition region.

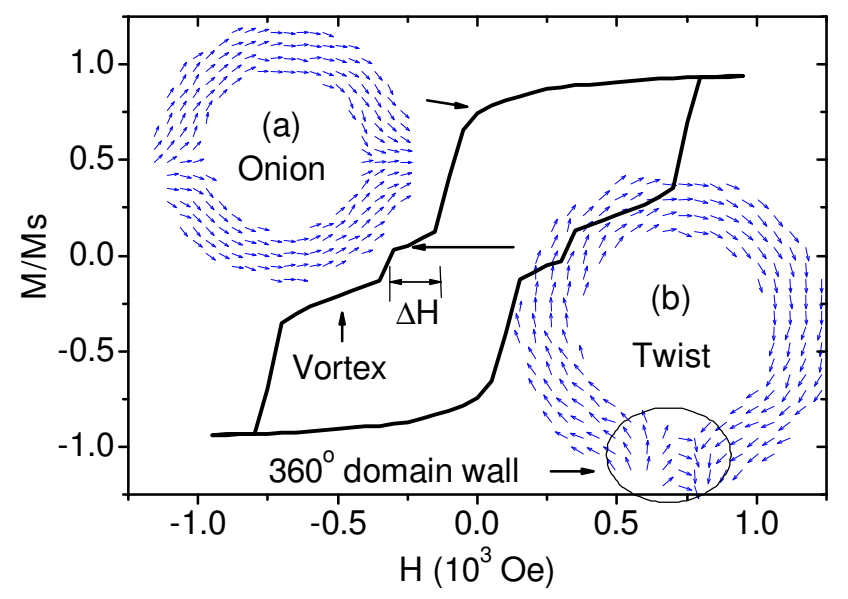

FIG. 5: (color online) Typical hysteresis curve for quadruple O-T-V-O switching ( $\left.R_{o}=150 \mathrm{~nm}, w=60 \mathrm{~nm}, h=15 \mathrm{~nm}\right)$. The insets (a) and (b) are snapshots during the transition. (a) is the onion state at remanence, while (b) is a typical twisted state, characterized by a $360^{\circ}$ domain wall.

\section{Quadruple Switching: O-T-V-O}

This switching process consists of four steps. In contrast to regular $\mathbf{O}-\mathbf{V}-\mathbf{O}$ triple switching, an additional step, characterized by the appearence of a twisted state (see Fig. 5 inset (b)), is involved. As mentioned above, twisted states are only found in rings with relatively small lateral dimensions 21 ( $\left(R_{o}<500 \mathrm{~nm}\right)$. In order to understand the conditions for the twisted state to occur, first recall the process of onion-to-vortex $(\mathbf{O}-\mathbf{V})$ transition during the regular triple switching: two domain walls move towards each other and annihilate by introducing a vortex core passing through the rim, i.e. entering from the outer edge and exiting through the inner edge. As the diameter of the vortex core is typically equal to $L_{e x}$, the width of the ring needs to be at least of the order of several exchange lengths in order to accommodate such a process. However, when the ring width is sufficiently small, the annihilation process is hindered. In that case, the two domain walls are stuck together, forming a $360^{\circ}$ domain wall (see Fig. 5 inset (b)). The configuration involving this $360^{\circ}$ domain wall is called "twisted state" 21 It turns out that this state can be stabilized within a fairly large field range $\Delta H$, e.g. for the specific ring shown in Fig. 5 it is stable between 100 Oe and 300 Oe. This result is consistent with previous literature $e^{21,23}$. Moveover, the results in Ref. 21 show precursor behavior of this type of switching, since the vortex state will not give positive magnetization when the magnetic field is negative.

From our simulations we find that the field range $\Delta H$ of the twisted state is sensitive to the geometric parameters $\left(R_{o}, w, h\right)$ in the following ways.

1. $h<L_{e x}$ : We find that the twisted state is not stable when the ring height is larger than $L_{e x}$. It has been reported that magnetic domain structures vary significantly along the vertical direction when the height is large $\underline{\underline{33}}$ Our simulations show that this is indeed the case when $h>L_{e x}$, as the exchange length $L_{e x}$ is a measure of typical domain size. In this case, the vertical stacking of slightly off-set domains eases the introduction of vortices in the annihilation process, destabilizing the $360^{\circ}$ domain wall. Furthermore, $\Delta H$ is most sensitive to ring height. The flatter the system, the larger $\Delta H$, i.e. $\Delta H$ can be doubled with decreased height. On the other hand, vortices cannot be formed if $h$ is too small $\left(<0.3 L_{e x}\right)$, which leads to the last switching process we will discuss in the next subsection.

2. $L_{e x}<w<5 L_{e x}$ : Typically, the field range $\Delta H$ varies with ring width (about 20\%), with a peak around $w=3 L_{e x}$. The reason for this is that there is a qualitative difference between thin and wide rings when the $360^{\circ}$ domain wall is destroyed. For rings with $w<L_{e x}$, the $360^{\circ}$ domain wall is not stable, with the exception of ultra-small rings $\left(R_{o}<2 L_{e x}\right)$. As it is the case for out-of-plane $\mathbf{O}$ $\mathrm{V}-\mathrm{O}$ switching, the spins in the domain area first point out-of-plane, and then turn in-plane along the field with increasing applied field. Slightly wider rings can support the $360^{\circ}$ domain wall in a small, but finite, field range and then evolve with the same process. For wider rings with $w>5 L_{e x}$, a vortex can be introduced through the outer edge of the ring and traverse through the rim, as already described for the $\mathbf{O}-\mathbf{V}-\mathbf{O}$ triple switching case. Slightly thinner rings still feature vortices. However, in this case the vortices cannot remain in the area of the ring where they entered when the reversed transverse field is increased. Instead, they move along the rim of the ring and finally 
exit. Hence, in this case the associated magnetization step in the hysteresis curve has a fairly large slope, indicating that the twisted region is moving with increasing applied field. The competition of these two mechanism gives rise to a peak of the field range where the twisted state is stable, which occurs at $w \approx 3 L_{e x}$.

3. $L_{e x}<R_{o} \lesssim 15 L_{e x}$ : In order to observe the twisted state, the entire ring has to be sufficiently small, but still larger than the single domain limit. The reason why larger rings destabilize the twisted state more easily is that in these structures vortices can enter from the outer rim. In smaller rings, vortices are not as easily accomodated in the domain wall region, because of differences between the arclengths of the outer and inner domain wall boundaries, due to the increased curvature. Another reason is that for large rings, there is a higher chance that defects serve as a catalyst of local vortex formation. $\Delta H$ is almost independent of $R_{o}$ for fixed aspect ratios $\left(\kappa=R_{i} / R_{o}\right)$. On the other hand, the width of the magnetization step in $M(H)$ where the vortex state is stable decreases quickly when $R_{o}$ is decreased, because the curvature eases the process of introducing a vortex during the $\mathbf{V}-\mathbf{O}$ process. When $\kappa$ is large and the ring is ultra-small, the extreme case where no vortex is involved emerges as discussed in detail below.

The above conditions are fulfilled in the gray regions of the phase diagrams in Fig. 1
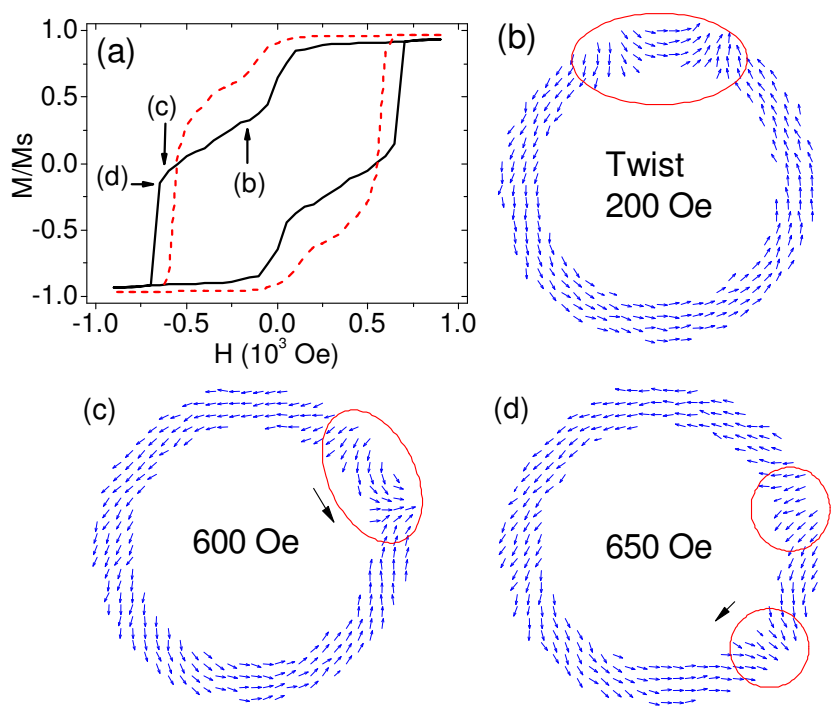

FIG. 6: (color online) Twisted triple switching (O-T-O): (a) Hysteresis curves for ring $1\left(R_{o}=70 \mathrm{~nm}, w=18 \mathrm{~nm}, h=5\right.$ $\mathrm{nm}$, black continuous line) and ring $2\left(R_{o}=42 \mathrm{~nm}, w=16\right.$ $\mathrm{nm}, h=14 \mathrm{~nm}$, red dashed line); (b) spin configuration for ring 1 at $H=200$ Oe; (c) spin configuration for ring 1 at $H=600 \mathrm{Oe}$; (d) spin configuration for ring 1 at $H=650$ Oe.

\section{Twisted triple switching (O-T-O)}

This process is an extreme limit of the $\mathbf{O}-\mathbf{T}-\mathbf{V}-\mathbf{O}$ switching process. Rather than forming a vortex state to lower the energy, the system chooses to move the entire domain wall towards either end of the ring along the magnetic field (see Fig. 6(c)), when the applied magnetic field is increased. Ultimately, the $360^{\circ}$ domain wall breaks up into two $180^{\circ}$ domain walls, and one of them travels back to the other side (see Fig. 6(d)), forming the reversed onion state. For relatively large rings, one can observe a small step in the magnetization curve, indicating the occurence of the twisted state (see Fig. 6(b)), while this step is not noticeable for very small rings. After the twisted state is formed, the magnetization decreases approximately linearly with increasing applied magnetic field, indicating the movement of the domain wall (see Fig. 6(c)). Finally, a large jump is observed, indicating the breakup (see Fig. 6(d)) of the $360^{\circ}$ domain wall and the formation of the reversed onion state. This process can only be observed under two extreme conditions:

1. Very small narrow rings (typically $R_{o}<3 L_{e x}$ and $\kappa>0.5$ ), when $0.3 L_{e x}<h<L_{e x}$ (see the small red region in Fig. 1(a)). The red dashed line in Fig 6 (a) is the typical hysteresis in this category. Here it is the curvature of the ring that prohibits the annihilation process. Under such conditions, the domain region typically covers a quarter of the ring.

2. Ultra-thin small narrow rings (typically $h<0.3 L_{e x}$ and $\kappa>0.5)$. The black solid line in Fig 6 (a) is the typical hysteresis. Fig 6(b)(c)(d) are snapshots of the configuration evolution with increasing applied magnetic field. In this case, it is the strong easy-plane shape anisotropy that hinders the annihilation process. For these ultra-thin systems, this switching process can be observed for rings as large as $R_{o} \sim 100 \mathrm{~nm}$ (see the fairly large red region in Fig प(c)). Theoretically they may exist on even larger rings. In reality, however, local defects will favor the formation of a local vortices, destroying the $360^{\circ}$ domain wall.

Note that the twisted state is stable at remanence once it is formed. Designs utilizing this state for magnetic memory have been proposed ${ }^{8}$. Our results show that such designs can use very small rings, for which there appears to be great potential to build high density storage systems.

\section{CONCLUSIONS}

In conclusion, we have computed the phase diagrams of magnetic nanorings, and identified three new types of magnetization reversal processes, such that there are now six known types of magnetization reversal processes in all 
reported for magnetic nanorings. The $\mathbf{O}^{\mathrm{V}}-\mathbf{V}-\mathbf{O}$ switching features a new out-of-plane onion state and easy switching process. Rings in this category have $h>w$, and $R_{o}$ can be as small as $L_{e x}$. The quadruple switching (O-T-V-O) and twisted triple switching (O-T-O) feature twisted states. Rings in these two categories are fairly small (typically with outer radius less than 200nm). Their width has to be less than about $4 L_{e x}$ to stabilize the $360^{\circ}$ domain wall. The field range where the twisted state is stable is most sensitive to the ring height. Small heights give rise to large field ranges, and there will be no twisted state when $h>L_{e x}$. We have combined our findings with results from previous literature into complete switching phase diagrams, shown in Fig.1. These should be helpful in designing magnetic nanorings with specific properties, and show the potential of building high density storage systems based on ring structures.

\section{ACKNOWLEDGEMENTS}

We would like to thank N.T. Jacobson and Y. Tao for useful discussions. Computing facilities were generously provided by the University of Southern California high-performance supercomputing center. We also acknowledge financial support by the Department of Energy under grant DE-FG02-05ER46240.
* Electronic address: shaas@usc.edu

1 K. De'Bell, A. B. Maclsaac, and J. P. Whitehead, Rev. Mod. Phys. 72(1), 225 (2000).

2 J. I. Martin, J. Nogues, J. L. V. K. liu, and I. K. Schuller, J. Magn. Magn. Mater. 256, 449 (2003).

3 R. P. Cowburn, J. Phys. D: Appl. Phys. 33, R1 (2001).

${ }^{4}$ M. Kläui, C. A. F. Vaz, L. Lopez-Diaz, and J. A. C. Bland, J. Phys.: Condens. Matter 15, R985 (2003).

${ }^{5}$ C. A. F. Vaz, T. J. Hayward, J. Llandro, F. Schackert, D. Morecroft, J. A. C. Bland, M. Kläui, and et al, J. Phys.: Condens. Matter 19, 255207 (2007).

6 J. G. Zhu, Y. Zheng, and G. A. Prinz, J. Appl. Phys. (2000).

7 J. Akerman, Science 308, 508 (2005).

${ }^{8}$ C. B. Muratov and V. V. Osipov, arxiv:0811.4663v1 (2008).

9 R. P. Cowburn and M. E. Welland, Science 287, 1466 (2000).

10 G. A. Prinz, Science 282, 1660 (1998).

11 M. M. Miller, G. A. Prinz, S. F. Cheng, and S. Bounnak, Appl. Phys. Lett. 81, 2211 (2002).

12 M. Kläui, J. Phys.: Condens. Matter 20, 313001 (2008).

13 W. Zhang, R. Singh, N. Bray-Ali, and S. Haas, Phys. Rev. B 77, 144428 (2008).

14 D. K. Singh, R. V. Krotkov, H. Xiang, T. Xu, T. P. Russell, and M. T. Tuominen, Nanotechnology 19, 245305 (2008).

15 G. D. Chaves-O'Flynn, A. D. Kent, and D. L. Stein, arxiv:0811.4440v1 (2008).

16 M. Beleggia, J. W. Lau, M. A. Schofield, Y. Zhu, S. Tandon, and M. DeGraef, J. Magn. Magn. Mater. 301, 131 (2006).

17 P. Landeros, J. Escrig, D. Altbir, M. Bahiana, and J. d'Albuquerque e Castro, J. Appl. Phys. 100, 044311 (2006).
18 M. Laufenberg, D. Backes, W. Buhrer, D. Bedau, M. Kläui, and et al, Appl. Phys. Lett. 88, 052507 (2006).

19 W. Zhang and S. Haas, arxiv:0902.3024v1 (2008).

20 M. Kläui, C. A. F. Vaz, L. J. Heyderman, and J. A. C. Bland, J. Magn. Magn. Mater. 290, 61 (2005).

21 F. J. Castano, C. A. Ross, C. Frandsen, A. Eilez, D. gil, H. I. . Smith, M. Redjdal, and F. B. Humphrey, Phys. Rev. B 67, 184425 (2003).

22 T. A. Moore, T. J. Hayward, D. H. Y. Tse, J. A. C. Bland, F. J. Castano, and C. A. Ross, J. Appl. Phys. 97, 063910 (2005).

23 D. K. Singh, R. V. Krotkov, and M. T. Tuominen, Phys. Rev. B 79, 184409 (2009).

24 J. d'Albuquerque e Castro, D. Altbir, J. C. Retamal, and P. Vargas, Phys. Rev. Lett. 88, 237202 (2002).

25 J. Mejia-Lopez, D. Altbir, A. H. Romero, X. Batlle, I. V. Roshchin, C. Li, and I. K. Schuller, J. Appl. Phys. 100, 104319 (2006).

26 J. Mejia-Lopez, P. Soto, and D. Altbir, Phys. Rev. B 71, 104422 (2005).

27 W. Zhang and S. Haas, J. Magn. Magn. Mater. 321, 3687 (2009).

28 N. Nowak, R. W. Chantrell, and E. C. Kennedy, Phys. Rev. Lett. 84, 163 (2000).

29 S. P. Li, D. Peyrade, M. Natali, A. Lebib, Y. Chen, U. Ebels, L. D. Buda, and K. Ounadjela, Phys. Rev. Lett. 86, 1102 (2001).

30 M. Steiner and J. Nitta, Appl. Phys. Lett. 84, 939 (2004).

31 M. Kläui and et al, Appl. Phys. Lett. 84, 951 (2004).

32 M. Steiner, G. Meier, U. Merkt, and J. Nitta, Physica E (2004).

33 M. Yan, R. Hertel, and C. M. Schneider, Phys. Rev. B 76, 094407 (2007). 